\section{Relationship between CD33 expression, splicing polymorphism, and in vitro cytotoxicity of gemtuzumab ozogamicin and the CD33/CD3 BiTE ${ }^{\circledR}$ AMG $\mathbf{3 3 0}$}

The CD33 antibody-drug conjugate gemtuzumab ozogamicin (GO) improves outcomes in some persons with acute myeloid leukemia (AML). ${ }^{1}$ Because GO is ineffective in many patients and/or may have adverse effects, there is interest in understanding factors, including CD33 expression, that possibly predict benefit and/or risk. A quantitative relationship between cell surface CD33 molecules and GO efficacy was demonstrable in engineered AML cell lines. ${ }^{2}$ However, validating this relationship clinically has been difficult. ${ }^{1}$ Still, CD33 expression, alternately estimated by mean fluorescence intensity [MFI] or the percentage of $\mathrm{CD} 33^{+}$blasts, has been associated with response to GO in several randomized trials. ${ }^{3-6}$

Recent efforts have centered on the relationship between GO benefit and CD33 genotypes. Most attention thus far has been paid to a single nucleotide polymorphism (SNP), rs12459419 (C>T), present in exon 2 close to the intron/exon junction. ${ }^{1}$ The minor $(\mathrm{T})$ allele results in reduced expression of full-length CD33 and preferential transcription of a variant missing exon 2 (CD33 $\varnothing \mathrm{E} 2)$ which is predicted to encode for a protein containing the $\mathrm{C} 2$-set but not $\mathrm{V}$-set domain. This is important because the V-set domain in the full-length CD33 protein contains the immune-dominant epitope(s) typically recognized by CD33 antibodies, including GO. In a large pediatric AML trial (COG-AAML0531), subjects with the rs 12459419 CC genotype (about $50 \%$ of study-entrants) had a significantly lower risk of relapse and better event- and disease-free survival when randomized to addition of GO whereas this benefit was not seen in patients with CT or TT genotypes, suggesting value of this SNP as response biomarker. However, uncertainties about the role of the rs12459419 genotype for GO-based therapy have remained, both with regard to prognostic significance (there was no evidence this
SNP was associated with response to GO in younger adults with AML treated on MRC/NCRI trials ${ }^{8}$ ) and mechanistically (we previously found forced overexpression of $\mathrm{CD}_{3} 3^{\triangle \mathrm{E} 2}$ in AML cells does not impact GOinduced cytotoxicity in vitro ${ }^{9}$ ). It is also unknown how the splicing polymorphism relates to the cytotoxic activity of other CD33-targeted drugs. These uncertainties prompted our studies examining the relationship of CD33 expression, rs12459419 genotype, and in vitro cytotoxic effects of GO and the CD33/CD3 Bispecific T-cell Engager (BiTE®) AMG 330, which also recognizes an epitope in CD33's V-set domain. ${ }^{10}$

Frozen aliquots of Ficoll-isolated mononuclear cells from pretreatment ("diagnostic") peripheral blood or bone marrow specimens from adults with AML were obtained from institutional AML cell repositories and cultured in vitro in short-term assays as described previously. ${ }^{11}$ We used the 2016 WHO criteria to define AML and the $2010 \mathrm{MRC/NCRI} \mathrm{criteria} \mathrm{to} \mathrm{assign} \mathrm{cytoge-}$ netic risk. Patients provided written informed consent for the collection and use of their specimens for research purposes under protocols approved by the Fred Hutchinson Cancer Research Center Institutional Review Board. Clinical data were de-identified in compliance with the Health Insurance Portability and Accountability Act. CD33 expression on primary AML samples was quantified with a PE-Cy7-labeled antibody (clone P67.6) as described. ${ }^{11}$ CD33 rs12459419 genotyping followed the methodology described by Gale et al. ${ }^{\circ}$ Specifically, gDNA was purified using Qiagen AllPrep DNA/RNA mini kit (Qiagen, Valencia, CA) and, typically, $200 \mathrm{ng}$ of gDNA amplified per reaction using the following primers (adapted from Gale et al.): For 5'-CTGGAAGCTGCTTCCTCAGACATG-3' and Rev 5'-GAACCAGTAACCATGAACTGGGGAG-3'. The resulting 266 bp CD33 amplicon was digested overnight with HaeIII (New England Biolabs, Ipswich, MA, USA) and separated on a $2 \%$ agarose gel.

To quantify drug-induced cytotoxicity, AML cells were incubated in 96-well round bottom plates at 5-10 x 103 cells/well with various concentrations of 1) GO (Pfizer,

Table 1. Patient and specimen characteristics, stratified by rs12459419 genotype.

\begin{tabular}{|c|c|c|c|c|}
\hline & All $(n=45)$ & $\Pi(n=5)$ & CT $(n=26)$ & CC $(n=14)$ \\
\hline Median age (range), years & $63.3(23.9-80.0)$ & $65.3(26.2-67.4)$ & $63.8(36.2-79.3)$ & $66.8(23.9-80.0)$ \\
\hline Female sex, n (\%) & $20(44.4 \%)$ & $3(60.0 \%)$ & $9(34.6 \%)$ & $8(57.1 \%)$ \\
\hline \multicolumn{5}{|l|}{ Cytogenetic risk } \\
\hline Favorable & $4(8.9 \%)$ & $1(20.0 \%)$ & $1(3.8 \%)$ & $2(14.3 \%)$ \\
\hline Intermediate & $30(66.7 \%)$ & $4(80.0 \%)$ & $18(69.2 \%)$ & $8(57.1 \%)$ \\
\hline Adverse & $11(24.4 \%)$ & $0(0.0 \%)$ & $7(26.9 \%)$ & $4(28.6 \%)$ \\
\hline \multicolumn{5}{|l|}{ Disease stage } \\
\hline Newly-diagnosed & $25(55.6 \%)$ & $2(40.0 \%)$ & $12(46.2 \%)$ & $11(78.6 \%)$ \\
\hline Relapsed/refractory & $20(44.4 \%)$ & $3(60.0 \%)$ & $14(53.8 \%)$ & $3(21.4 \%)$ \\
\hline \multicolumn{5}{|l|}{ Specimen source } \\
\hline Bone marrow & $22(48.9 \%)$ & $2(40.0 \%)$ & $13(50.0 \%)$ & $7(50.0 \%)$ \\
\hline Peripheral blood & $23(51.1 \%)$ & $3(60.0 \%)$ & $13(50.0 \%)$ & $7(50.0 \%)$ \\
\hline Median \% blasts (range) & $81.1(27.9-97.0)$ & $83.8(58.4-92.2)$ & $86.7(27.9-97.0)$ & $83.0(58.7-95.5)$ \\
\hline Median \% T cells (range) & $2.8(0.04-27.3)$ & $2.3(1.2-7.2)$ & $2.4(0.2-27.3)$ & $3.3(0.04-11.4)$ \\
\hline Median \% viability at thaw (range) & $93.8(24.3-99.3)$ & $88.2(24.3-94.4)$ & $93.0(61.4-99.3)$ & $95.9(26.9-99.1)$ \\
\hline Median \% viability at 48 hours (range) & $71.8(13.4-93.5)$ & $82.5(37.8-91.4)$ & $74.2(21.9-93.5)$ & $69.7(13.4-93.2)$ \\
\hline
\end{tabular}


New York, NY) or 2) AMG 330 (kindly provided by Amgen, Amgen Research $\mathrm{GmbH}$, Munich, Germany) alone or together with healthy-donor $\mathrm{T}$ cells (labeled with CellVue Burgundy dye) at an effector-to-target (E:T) cell ratio of $1: 1$ as described. ${ }^{9,12}$ After 2 (AMG 330) or 3 (GO) days, cell numbers and drug-induced cytotoxicity, using 4',6-diamidino-2-phenylindole (DAPI) to detect non-viable cells, were determined flow cytometrically. Linear median fluorescence intensity (MFI) values were used to quantify CD33 expression levels. Drug-specific cytotoxicity is presented as: \% cytotoxicity $=100 \times(1-$ live target cells treated $_{\text {/ive target cells }}$ control $)$. Results are presented as mean \pm SEM. ${ }^{12}$ Statistical analyses were performed with Prism 7 (Graphpad; La Jolla, CA, USA).

We had pre-treatment samples available from 45 adults with AML for our analyses (Table 1). Among these, genotypes were CC $(n=14[31.1 \%])$, CT $(n=26[57.8 \%])$, and TT $(n=5[11.1 \%])$. While the proportion of samples with
TT genotype was similar to that in the $>1,000$ people enrolled in COG-AAML05317 and the 720 participants of MRC/NCRI trials, ${ }^{8}$ our cohort, likely due to the smaller sample size, contained a higher proportion of samples with CT and lower proportion of samples with CC genotypes than the COG-AAML0531 and MRC/NCRI cohorts. In line with data from the COG-AAML0531 and MRC/NCRI cohorts, cell surface display of CD33 was highest in people with CC genotype (MFI: 2,344 \pm 579 ) and lowest in those with TT genotype (255 \pm 91$)$, with people with CT genotype having intermediate expression $(888 \pm 137 ; P=0.0035$ [Kruskal-Wallis test]; Figure 1a); CD33 expression data stratified by rs12459419 genotype, cytogenetic risk, and disease stage are summarized in Online Supplementary Table S1. In our specimen series, we found a linear relationship between CD33 expression and $\% \mathrm{CD}_{3}{ }^{+}$blasts $\left(\mathrm{r}^{2}=0.8159 ; P<0.0001\right.$ [automated elimination of 2 outlier values] and, consequently, lower
A

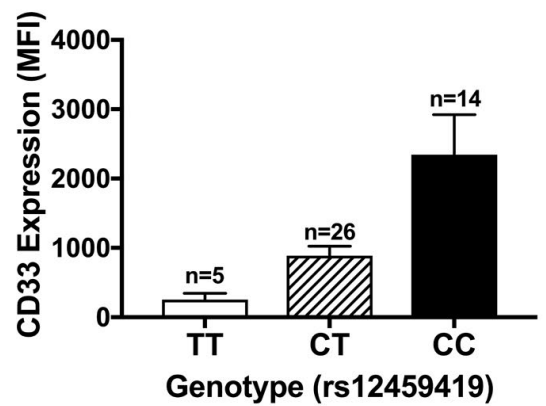

B

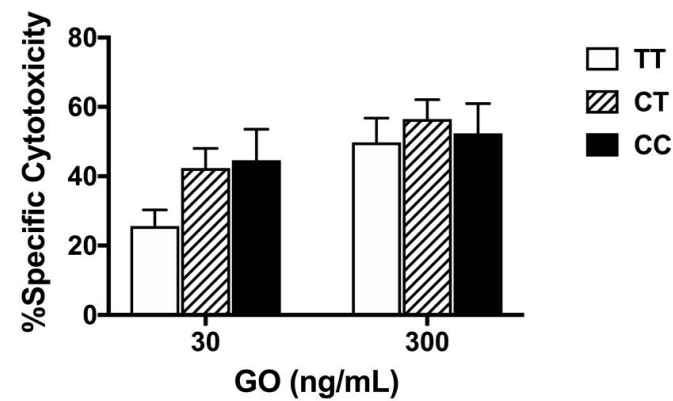

C
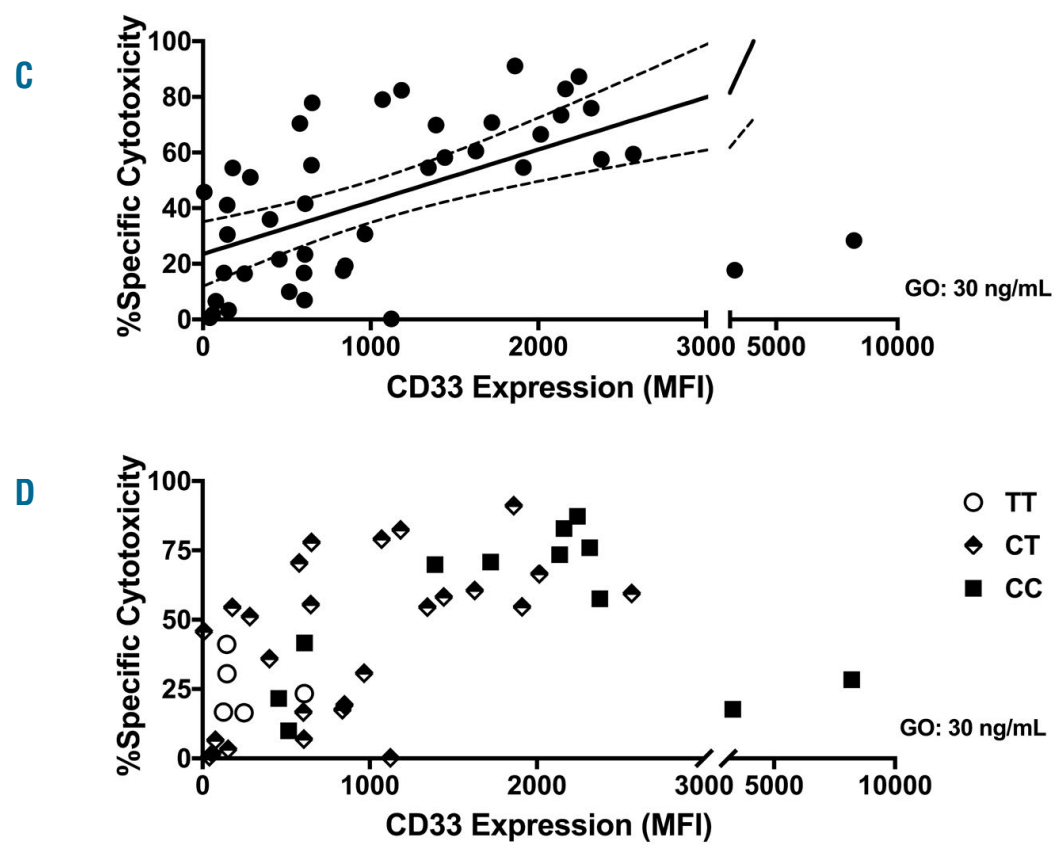

Figure 1. Relationship between cell surface expression of CD33, CD33 genotype (rs12459419), and in vitro G0-induced cytotoxicity in primary AML samples. (A) Upon thaw, Ficoll-isolated mononuclear cells from pretreatment peripheral blood or bone marrow specimens from adults with AML were stained with directly labeled antibodies recognizing CD33 (clone P67.6), CD3, and CD45, among others, and analyzed by flow cytometry. AML blasts were identified by CD45/sidescatter properties. (B) Aliquots of primary AML cells were incubated with one of two doses of GO (corresponding to 30 or $300 \mathrm{ng} / \mathrm{mL}$ calicheamicin) for 3 days, followed by determination of cell numbers and drug-induced cytotoxicity, using DAPI to detect non-viable cells. As point of reference, peak plasma concentrations of $79 \pm 93 \mathrm{ng} / \mathrm{mL}$ (mean \pm standard deviation) of total calicheamicin have been reported in patients with relapsed AML treated with a single $9 \mathrm{mg} / \mathrm{m} 2$ dose of G0.14 AML cells were identified by forward/side scatter properties. (C) Linear regression between CD33 expression and drug-induced cytotoxicity (GO/calicheamicin dose: $30 \mathrm{ng} / \mathrm{mL}$ ). Dashed line: $95 \%$ confidence interval. (D) Relationship between cytotoxicity induced by $30 \mathrm{ng} / \mathrm{mL}$ of GO/calicheamicin and CD33 expression, stratified by rs12459419 genotype. MFI: mean fluorescence intensity. 
percentages of $\mathrm{CD}_{3} 3^{+}$blasts in individuals with TT than $\mathrm{CT}$ and TT genotypes (Online Supplementary Figure S1A and $B)$.

For all 45 samples, material was available to perform in vitro cytotoxicity assays with $\mathrm{GO}$. At both tested doses (equivalent to $30 \mathrm{ng} / \mathrm{mL}$ and $300 \mathrm{ng} / \mathrm{mL}$ of calicheamicin), significant drug-induced cytotoxicity was found across rs12459419 genotypes (Figure 1B). At the lower GO dose, drug-induced cytotoxicity was less marked in TT than CC/CT samples. Acknowledging the limitations of a relatively small sample size, however, there were no statistically significant differences in the percentage of specific cytotoxicity induced by GO between specimens with TT, CT, and CC genotypes. Still, we found a relationship between CD33 expression and GO-induced cytotoxicity as indicated by linear regression analysis $\left(r^{2}=0.3124 ; P<0.0001\right.$ [automated elimination of 2 outlier values]; Figure 1c). Conversely, for any given level of CD33 expression (or \%CD33+ blasts), there was no evidence of greater GO sensitivity of samples with rs12459419 CC genotype compared to those with CT or TT genotype (Figure 1D; Online Supplementary Figure S1C for data on $\% \mathrm{CD} 33^{+}$blasts). Together, these findings indicate that the in vitro sensitivity of leukemia cells is impacted by the amount of CD33 expressed on the cell surface, whereas the rs12459419 genotype per se did not influence GO-sensitivity to a measurable degree. In COG-AAML0531, multivariable analyses which included CD33 genotype, cytogenetic/molecular risk and cell surface CD33 levels showed that the rs12459419 CC genotype is independently associated with fewer relapses and better disease-free survival displacing CD33 density as a

A

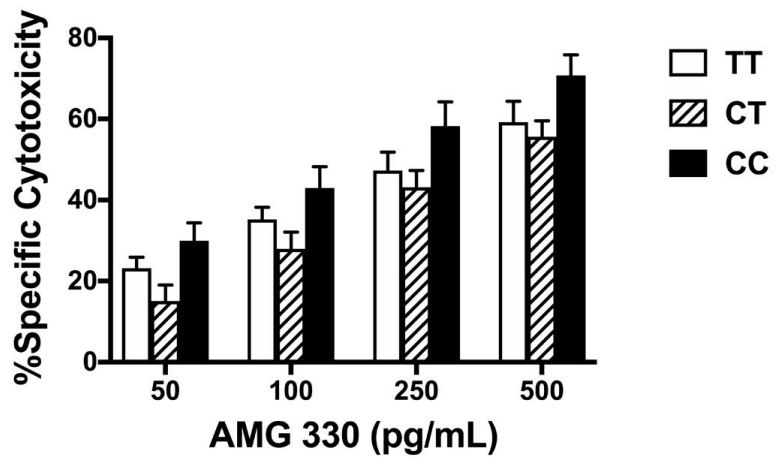

B

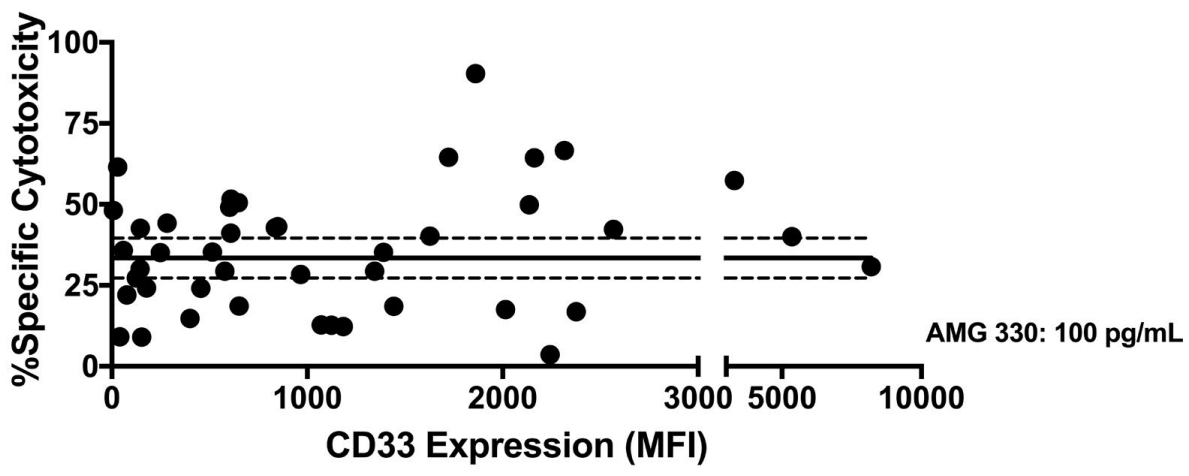

C

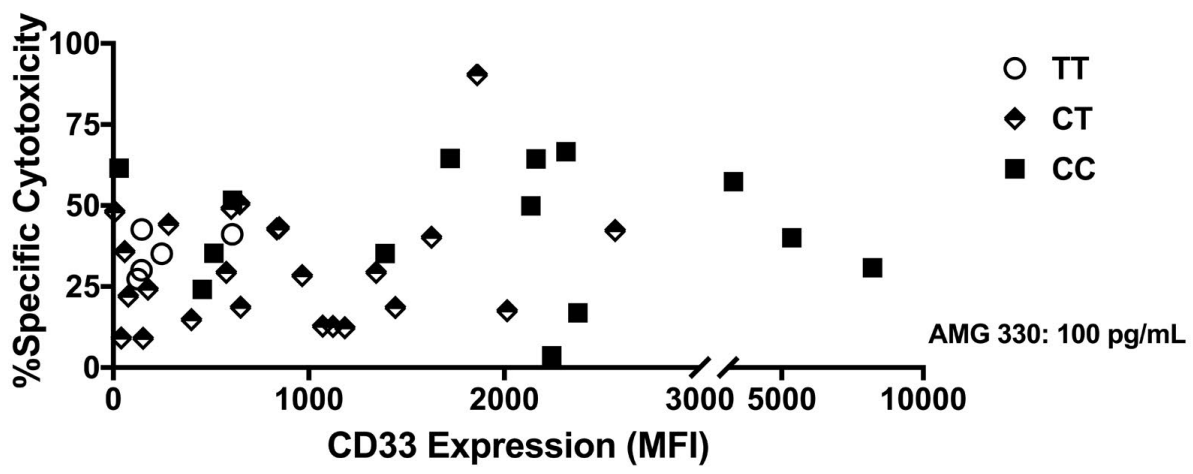

Figure 2. Relationship between cell surface expression of CD33, CD33 genotype (rs12459419), and in vitro AMG 330-induced cytotoxicity in primary AML samples. (A) Aliquots of primary AML cells were incubated with various doses of AMG 330 as indicated together with healthy-donor T cells at an E:T cell ratio of 1:1. As point of reference, steady state concentrations of $621 \pm 502 \mathrm{pg} / \mathrm{mL}$ (mean \pm standard deviation) have been reported in patients with relapsed/refractory B-acute lymphoblastic leukemia treated with $28 \mu \mathrm{g} /$ day of the CD19/CD3 BiTE blinatumomab. ${ }^{15}$ After 2 days, cell numbers and drug-induced cytotoxicity were determined using DAPI to detect non-viable cells. AML cells were identified by forward/side scatter properties and negativity for CellVue Burgundy dye. (B) Linear regression between CD33 expression and drug-induced cytotoxicity (AMG 330 dose: 100 pg/mL). Dashed line: 95\% confidence interval. (C) Relationship between cytotoxicity induced by 100 pg/mL of AMG 330 and CD33 expression, stratified by rs12459419 genotype. MFI: mean fluorescence intensity. 
prognostic variable. ${ }^{7}$ Since our in vitro studies demonstrate that leukemia cells with rs12459419 CT and TT genotype are not intrinsically resistant to GO, other causes will need to be considered to account for the lack of GO benefit with these 2 genotypes in COG-AAML0531.

Stimulated by the success and limitations of GO, several new CD33-targeting therapeutics, including AMG 330 , have entered clinical testing. ${ }^{13}$ We previously reported a linear relationship between cell surface CD33 abundance and AMG 330 cytotoxicity in engineered cell lines. ${ }^{12}$ However, in a larger series of primary AML specimens which encompassed all 45 specimens included in this study, such a relationship was only seen at higher $\mathrm{E}: T$ cell ratios and higher drug doses, ${ }^{11}$ possibly indicating cell surface CD33 is a less critical parameter for AMG 330 than GO. As shown in Figure 2A, across the tested AMG 330 doses (50-500 pg/mL), significant cytotoxicity was found in the presence of healthy donor $\mathrm{T}$ cells in all rs12459419 genotypes. Similar to the data obtained with $\mathrm{GO}$, there were no statistically significant differences in the percentage of AMG 330-induced specific cytotoxicity between specimens with TT, CT, and CC genotypes. Consistent with our previous analyses, there was no linear relationship between CD33 expression and AMG 330 -induced cytotoxicity (at $100 \mathrm{pg} / \mathrm{mL} ; \mathrm{r}^{2}=0.0136$, $P=0.45$; Figure $2 \mathrm{~B}$ ) at this relatively low E:T ratio. Moreover, for any given level of CD33 expression (or $\% \mathrm{CD}_{3} 3^{+}$blasts), there was no evidence of greater AMG 330 sensitivity of samples with rs12459419 CC genotype compared to those with CT or TT genotype (Figure 2C; Online Supplementary Figure S1D for data on $\% \mathrm{CD}_{3} 3^{+}$ blasts). Similar results were obtained when examining AMG 330-induced cytotoxicity without exogenous healthy-donor $\mathrm{T}$ cells or when restricting analyses to samples from adults with newly-diagnosed AML or those from individuals with relapsed/refractory AML (Online Supplementary Figures S2-4).

In summary, we found no evidence that the cytotoxic properties of GO or AMG 330 are substantially affected by the rs12459419 genotype per se. Rather, for GO, cell surface abundance of CD33 was associated with druginduced cytotoxicity, possibly as a (likely imperfect) surrogate for intracellular drug delivery. In contrast, we found no evidence that CD33 expression levels significantly correlated with AMG 330 cytotoxicity. The latter suggests that even limited amounts of CD33 allow efficient immune synapse formation with $\mathrm{T}$ cells and may point to a conceptual advantage of using bispecific antibodies over GO to eliminate leukemia cells with limited CD33 display.

George S. Laszlo, 1 Mary E. Beddoe, ${ }^{1}$ Colin D. Godwin, ${ }^{2}$

Olivia M. Bates, ${ }^{1}$ Chelsea J. Gudgeon,

Kimberly H. Harrington ${ }^{1}$ and Roland B. Walter ${ }^{2,34}$

GSL and MED contributed equally to this work.

${ }^{1}$ Clinical Research Division, Fred Hutchinson Cancer Research Center; ${ }^{2}$ Hematology/Oncology Fellowship Program; ${ }^{3}$ Department of Medicine, Division of Hematology and ${ }^{4}$ Department of Epidemiology, University of Washington, Seattle, WA, USA

Funding: this research was funded in part through the National Institutes of Health/National Cancer Institute (NIH/NCI) Cancer
Center Support Grant (P30-CA015704) and a sponsored research agreement with Amgen, Inc. (to RBW). CDG is supported by a fellowship training grant from the National Heart, Lung, and Blood Institute (NHLBI)/NIH (T32-HL007093). RBW is a Leukemia \& Lymphoma Society Scholar in Clinical Research.

Correspondence: rwalter@fredhutch.org doi:10.3324/haematol.2018.202069

Information on authorship, contributions, and financial \& other disclosures was provided by the authors and is available with the online version of this article at www. haematologica.org.

\section{References}

1. Godwin CD, Gale RP, Walter RB. Gemtuzumab ozogamicin in acute myeloid leukemia. Leukemia. 2017;31(9):1855-1868.

2. Walter RB, Raden BW, Kamikura DM, Cooper JA, Bernstein ID. Influence of CD33 expression levels and ITIM-dependent internalization on gemtuzumab ozogamicin-induced cytotoxicity. Blood. 2005;105(3):1295-1302

3. Amadori S, Suciu S, Selleslag D, et al. Gemtuzumab ozogamicin versus best supportive care in older patients with newly diagnosed acute myeloid leukemia unsuitable for intensive chemotherapy: results of the randomized phase III EORTC-GIMEMA AML-19 trial. J Clin Oncol. 2016;34(9):972-979.

4. Olombel G, Guerin E, Guy J, et al. The level of blast CD33 expression positively impacts the effect of gemtuzumab ozogamicin in patients with acute myeloid leukemia. Blood. 2016;127(17):21572160.

5. Khan N, Hills RK, Virgo P, et al. Expression of CD33 is a predictive factor for effect of gemtuzumab ozogamicin at different doses in adult acute myeloid leukaemia. Leukemia. 2017;31(5):1059-1068.

6. Pollard JA, Loken M, Gerbing RB, et al. CD33 expression and its association with gemtuzumab ozogamicin response: results from the randomized phase III Children's Oncology Group trial AAML0531. J Clin Oncol. 2016;34(7):747-755.

7. Lamba JK, Chauhan L, Shin M, et al. CD33 splicing polymorphism determines gemtuzumab ozogamicin response in de novo acute myeloid leukemia: report from randomized phase III Children's Oncology Group trial AAML0531. J Clin Oncol. 2017;35(23):26742682.

8. Gale RE, Popa T, Wright M, et al. No evidence that CD33 splicing SNP impacts the response to GO in younger adults with AML treated on UK MRC/NCRI trials. Blood. 2018;131(4):468-471.

9. Laszlo GS, Harrington KH, Gudgeon CJ, et al. Expression and functional characterization of CD33 transcript variants in human acute myeloid leukemia. Oncotarget. 2016;7(28):43281-43294.

10. Friedrich $M$, Henn $A$, Raum $T$, et al. Preclinical characterization of AMG 330, a CD3/CD33-bispecific T-cell-engaging antibody with potential for treatment of acute myelogenous leukemia. Mol Cancer Ther. 2014;13(6):1549-1557

11. Harrington KH, Gudgeon CJ, Laszlo GS, et al. The broad anti-AML activity of the CD33/CD3 BiTE antibody construct, AMG 330, is impacted by disease stage and risk. PLoS One. 2015;10(8):e0135945.

12. Laszlo GS, Gudgeon CJ, Harrington KH, et al. Cellular determinants for preclinical activity of a novel CD33/CD3 bispecific T-cell engager (BiTE) antibody, AMG 330, against human AML. Blood. 2014;123(4):554-561.

13. Walter RB. Investigational CD33-targeted therapeutics for acute myeloid leukemia. Expert Opin Investig Drugs. 2018;27(4):339-348.

14. Dowell JA, Korth-Bradley J, Liu H, King SP, Berger MS Pharmacokinetics of gemtuzumab ozogamicin, an antibody-targeted chemotherapy agent for the treatment of patients with acute myeloid leukemia in first relapse. J Clin Pharmacol. 2001 Nov;41(11):1206-1214.

15. Zhu M, Kratzer A, Johnson J, et al. Blinatumomab pharmacodynamics and exposure-response relationships in relapsed/refractory acute lymphoblastic leukemia. J Clin Pharmacol. 2018;58(2):168-179. 